Research Paper

\title{
Fatty Acid Synthase Mediates the Epithelial- Mesenchymal Transition of Breast Cancer Cells
}

\author{
Junqin $\mathrm{Li}^{1}$, Lihua Dong${ }^{1}$, Dapeng Wei ${ }^{1}$, Xiaodong Wang ${ }^{2}$, Shuo Zhang ${ }^{1}$, Hua Li ${ }^{1}$ \\ 1. Department of Basic and Forensic Medicine, Sichuan University, Sichuan Province, 610041, China. \\ 2. West China Hospital, Sichuan University, Sichuan Province, 610041, China.
}

$\triangle$ Corresponding author: Hua Li, West China College of Basic and Forensic Medicine, Sichuan University, 17 People's South Road, Chengdu, Sichuan Province, 610041, China. E-mail address: lihua_scu@scu.edu.cn.

(c) Ivyspring International Publisher. This is an open-access article distributed under the terms of the Creative Commons License (http://creativecommons.org/ licenses/by-nc-nd/3.0/). Reproduction is permitted for personal, noncommercial use, provided that the article is in whole, unmodified, and properly cited.

Received: 2013.08.06; Accepted: 2013.12.09; Published: 2014.01.20

\begin{abstract}
This study aimed to investigate the role of fatty acid synthase (FASN) in the epithelial-mesenchymal transition (EMT) of breast cancer cells. MCF-7 cells and MCF-7 cells overexpressing mitogen-activated protein kinase 5 (MCF-7-MEK5) were used in this study. MCF-7-MEK5 cells showed stable EMT characterized by increased vimentin and decreased E-cadherin expression. An In vivo animal model was established using the orthotopic injection of MCF-7 or MCF-7-MEK5 cells. Real-time quantitative PCR and western blotting were used to detect the expression levels of FASN and its downstream proteins liver fatty acid-binding protein (L-FABP) and VEGF/VEGFR-2 in both in vitro and in vivo models (nude mouse tumor tissues). In MCF-7-MEK5 cells, significantly increased expression of FASN was associated with increased levels of L-FABP and VEGF/VEGFR-2. Cerulenin inhibited MCF-7-MEK5 cell migration and EMT, and reduced FASN expression and down-stream proteins L-FABP, VEGF, and VEGFR-2. MCF-7-MEK5 cells showed higher sensitivity to Cerulenin than MCF-7 cells. Immunofluorescence revealed an increase of co-localization of FASN with VEGF on the cell membrane and with L-FABP within MCF-7-MEK5 cells. Immunohistochemistry further showed that increased percentage of FASN-positive cells in the tumor tissue was associated with increased percentages of L-FABP- and VEGF-positive cells and the Cerulenin treatment could reverse the effect. Altogether, our results suggest that FASN is essential to EMT possibly through regulating L-FABP, VEGF and VEGFR-2. This study provides a theoretical basis and potential strategy for effective suppression of malignant cells with EMT.
\end{abstract}

Key words: EMT; FASN; L-FABP; VEGF; Breast cancer.

\section{Introduction}

Breast cancer accounts for $7 \%-10 \%$ of all malignant tumors in women. However, its pathogenesis remains elusive. Metastasis and drug resistance, two key obstacles to cure breast cancer, are related to the epithelial-mesenchymal transition (EMT). In tumors, epidermal cells gain characteristics of mesenchymal cells via EMT, such as metastasis, self-renewal capacity, and increased resistance to conventional therapies [1]. Hence, searching for factors that effectively impact EMT could be a strategy not only to combat cancer metastasis but also to conquer drug resistance.

Fatty acid synthase (FASN) is a key enzyme required for the anabolic conversion of dietary carbohydrates to fatty acids and functions normally in cells with high lipid metabolism. Cells with EMT show faster proliferation, and fatty acid metabolism in these cells changes correspondingly [2]. Hung et al. [3] found that FASN inhibitor osthole could abrogate hepatocyte growth factor (HGF)-induced EMT, including cell scattering, migration, and invasion in MCF-7 breast cancer cells.

Vascular endothelial growth factor (VEGF) stimulates the formation of new blood vessels [4]. Gonzalez-Moreno et al. [5] have found that increased 
expression of VEGF in malignant cells during the transition from prostate intraepithelial neoplasia (PIN) to invasive carcinoma leads to EMT. VEGF stimulates multiple cellular responses by interacting with classic, tyrosine kinase VEGF receptors, VEGFR-1 (flt-1) and VEGFR-2 (KDR, flk-1). Surprisingly, the relative expression of these tyrosine kinase receptors, especially VEGFR-2 (KDR), on breast carcinoma cells and their putative functions have not been resolved adequately ${ }^{[6]}$.

The mammalian fatty acid-binding proteins (FABPs) bind to long chain fatty acids (FA). Multiple studies have shown that individual FABPs possess both unique and overlapping functions [7]. Liver fatty acid binding protein (L-FABP) is the only FA-binding member of the mammalian FABP family that transfers FA to membranes by aqueous diffusion. L-FABP binds to long chain saturated FA, the main products of FASN, with higher affinity than other FAs ${ }^{[8]}$. Previous study has shown that L-FABP is significantly up-regulated in clear cell renal cell carcinoma (CCRCC) through EMT [9].

MEK5/Erk5 has been shown a pivotal role in the MAPK signaling pathway. Zhou et al. reported that overexpression of MEK5 in MCF-7 breast cancer cells could induce EMT and TNF-a resistance [10]. In our previous experiment we observed that MCF-7-MEK5 cells showed increased expressions of FASN, L-FABP and VEGF/VEGFR compared to MCF-7 cells. FASN over-expression in breast cancer cells might result in excessive synthesis of long-chain FAs that are transferred to the cell membrane by L-FABP and regulate the expression of membrane proteins such as VEGFR2 by affecting their localization through lipid rafts [11]. In this way, the expressions of its ligand VEGF and downstream MEK5 could be promoted and EMT could be induced in the cells.

In the present study, we used an inhibitor of FASN, Cerulenin, to investigate the role of FASN and Cerulenin's interaction with L-FABP and VEGF/VEGFR in MCF-7-MEK5 cells with EMT and also in nude mouse tumor tissues.

\section{Materials and Methods}

\section{Cell lines and cultures}

The human breast cancer cell lines (MCF-7 and MCF-7-MEK) were gifts from the Immunology Laboratory of West China Medical Center, Sichuan University, and cultured in a RPMI-1640 medium containing $10 \%$ fetal bovine serum under $5 \%$ $\mathrm{CO}_{2} / 95 \%$ in a $\mathrm{CO}_{2}$ incubator. Cell culture medium was obtained from Invitrogen (Burlington, ON, Canada).

\section{Establishment of breast cancer orthotopic injection model}

Eighteen specific-pathogen-free BALB/c nude mice (5 weeks, female, weighed 18-20 g) were purchased from the Experimental Animal Center of Sichuan University. The animals were housed and handled in accordance with approved guidelines by the animal welfare committee of Sichuan province (Protocol number: SYXK [Sichuan] 2009-045). All mice were randomly divided into the MCF-7 group and MCF-7-MEK5 group with 9 mice each. For each group 6 mice (experimental subgroup) received cell injections and 3 mice received solvent as a control. MCF-7 and MCF-7-MEK5 cells $\left(1 \times 10^{7} / \mathrm{ml}\right)$ were orthotopically injected under the breast pads $(0.1 \mathrm{ml} /$ mouse). When the tumor grew to the size of $100 \mathrm{~mm}^{3}$, Cerulenin (BML-G237-0025, Biomol) was administered by intraperitoneal injection at $160 \mathrm{mg} / \mathrm{d} / \mathrm{kg}$ for $10 \mathrm{con}-$ secutive days. Five days later, mice were killed and the tumor models were collected.

\section{Cell viability assay}

Cells $\left(5 \times 10^{4} / \mathrm{ml}\right)$ were cultured in 96-well plates. Twenty-four hours later, cells were treated with a serial concentrations of Cerulenin ( 0 to $80 \mu \mathrm{g} / \mathrm{ml})$ for $24 \mathrm{~h}$. Cells were then incubated with MTT $(5 \mathrm{mg} / \mathrm{ml}$, $10 \mu \mathrm{l} /$ well) for $4 \mathrm{~h}$ and centrifuged. After removal of supernatants, DMSO was added (100 $\mu \mathrm{l} /$ well $)$ and the plates were shaken in dark for $10 \mathrm{~min}$. Absorption value was determined by automatic microplate reader (Bio-Rad, Hercules, CA, USA) at A570. Cell viability = (A570 Drug Treated Group/ A570 Control Group) $\times 100 \%$.

\section{Wound healing assay}

Cells $\left(4.5 \times 10^{5} / \mathrm{mL}\right)$ were plated in 24 -well dishes and incubated overnight to yield confluent monolayers for wound healing assay. Wounds were made in cells with or without Cerulenin using a pipette tip. Photographs were taken immediately (time zero) and $24 \mathrm{~h}$ after wounding. The distance of cell monolayer migration toward the wounded area was measured. The result was expressed as a migration index $=$ the distance of the Cerulenin-treated group / the distance of the control group cells.

\section{Western blotting (WB) assay}

Protein was extracted by Lysis buffer (KGP2100, KeyGEN BioTECH) and the concentration of protein was measured by bicinchoninic acid protein assay kit (KGSK3051, KeyGEN BioTECH). Fifty micrograms of protein was resuspended in sample loading buffer, boiled for $5 \mathrm{~min}$, and electrophoresed on polyacrylamide gel. The proteins were transferred electrophoretically to a nitrocellu-lose membrane, blocked with 
PBS-Tween $(0.05 \%)$ in $5 \%$ low-fat dry milk solution at room temperature for $2 \mathrm{~h}$, and incubated with specific antibodies FASN (1:5000, 273kDa, ab22759, abcam), L-FABP (1:1000, 15kDa; ab7807, abcam), VEGF (1:500, 45kDa; bs-0279R, Bioss), VEGFR-2 (1:500, 151kDa; bs-0565R, Bioss), MEK5 (1:500, 49kDa; bs-4124R, Bioss), E-cadherin (1:500, 80kDa; bs-1016R, Bioss), or vimentin (1:500, $51 \mathrm{kDa}$; bs-0756R, Bioss) overnight at $4^{\circ} \mathrm{C}$. The horseradish peroxidase-labeled goat-anti-rabbit (ZB 2301) or goat-anti-mouse (ZSGB-BIO) secondary antibodies were incubated at room temperature for $2 \mathrm{~h}$. ECL (KGP1121, KeyGEN BioTECH) was used for chemiluminescence color reaction.

\section{Real-time PCR}

Total RNA was extracted from cells/tissues by Tripure (1667165001, Roche). The reverse transcription was carried out with a SuperScript first-strand synthesis system (Invitrogen) using Oligo (dT) 12-18 primers (\#K1621, Fermentas). Specific primer used to amplify the genes FASN, L-FABP, VEGF, VEGFR2, MEK5, E-cadherin, vimentin (Table 1, primer pairs used for RT-PCR) was designed using online software Primer 3 (V. 0.4.0, Whitehead Institute, USA), and the endogenous GAPDH was employed as an internal standard. Primer specificity was confirmed by Basic Local Alignment Search Tool (BLAST), and the primers were synthesized by Shanghai Bioengineering Co. Standard PCR was performed using a PCR kit with Taq (D7232, Beyotime). The PCR products were isolated by $1.5 \%$ agarose gel electrophoresis, and the bands were visualized by Goldview (G8142, Solarbio) staining. Real-time PCR was performed according to the standard procedures using Maxima SYBR Green/ROX qPCR Master Mix (2X) kit (\#k0221, Fermentas). Primer annealing temperatures were all $60^{\circ} \mathrm{C}$. Data were analyzed with a normalized gene expression method $(\Delta \Delta \mathrm{Ct})$ using the iQ5 Optical System Software (BioRad).

\section{Immunofluorescence cytochemistry assay}

Slides with monolayer cells were fixed with $4 \%$ paraformaldehyde for $30 \mathrm{~min}$. After air dried, they were permeabilitied with $0.25 \%$ Triton X-100 for 30 min, incubated with specific primary antibodies (FASN, 1:100; L-FABP, 1:50; VEGF, 1:30) at $4^{\circ} \mathrm{C}$. Then secondary antibodies were added (anti-mouse IgG, anti-rabbit IgG CY3 conjugate antibody developed in sheep: Sigma) for another incubation at $37^{\circ} \mathrm{C}$ for 30 min. The nucleus was stained using DAPI. Digital images were captured using a Nikon Eclipse 80i microscope along with the accompanying program NIS-Element F. Semi-quantitative analysis of fluorescent image was performed using Image J (National
Institute of Health, USA). Co-localization signal was determined by setting a threshold in the gray images using Image J.

Table I. Primer pairs used in RT-PCR.

\begin{tabular}{lllc}
\hline $\begin{array}{l}\text { Gene } \\
\text { Name }\end{array}$ & & Primer Sequence $\left(5^{\prime} \rightarrow 3^{\prime}\right)$ & $\begin{array}{c}\text { Product } \\
\text { Length }(\mathrm{bp})\end{array}$ \\
\hline GAPDH & $\mathrm{F}$ & CTGCCCCTCTGCTGATG & 150 \\
& $\mathrm{R}$ & TCCACGATACCAAAGTTGTCAT & \\
FASN & $\mathrm{F}$ & CTTCCGAGATTCCATCCTACGC & 131 \\
& $\mathrm{R}$ & TGGCAGTCAGGCTCACAAACG & \\
L-FABP & $\mathrm{F}$ & ACTGCAGAGCCAGGAAAACT & 162 \\
& $\mathrm{R}$ & TCGTTTTGATCACTTTGGA & \\
VEGFA & $\mathrm{F}$ & GAAAGGGGCAAAAACGAAAG & 128 \\
& $\mathrm{R}$ & TTTGCAGGAACATTACACGTC & \\
VEGFR2 & $\mathrm{F}$ & AGTGATCGGAAATGACACTGG & 135 \\
& $\mathrm{R}$ & TACACGACTCCATGTTGGTCA & \\
E-cadherin & $\mathrm{F}$ & AACGCATTGCCACATACACTC & 185 \\
& $\mathrm{R}$ & GACCTCCATCACAGAGGTTCC & \\
Vimentin & $\mathrm{F}$ & TCAATGTTAAGATGGCCCTTG & 155 \\
& $\mathrm{R}$ & TGAGTGGGTATCAACCAGAGG & \\
MEK5 & $\mathrm{F}$ & CCAGAACATGTCCTTGGAAGA & 162 \\
& $\mathrm{R}$ & CACCAGCTGAGTGCTAACTCC & \\
\hline
\end{tabular}

\section{Immunohistochemistry assay}

Tumor tissues obtained from nude mice were fixed immediately with $4 \%$ paraformaldehyde for 2 days followed by embedded with paraffin and sectioned $(4 \mu \mathrm{m})$ onto the slides. The sections were baked at $60^{\circ} \mathrm{C}$ for $2 \mathrm{~h}$, deparaffinized by two changes of $\mathrm{xy}-$ lene, and rehydrated in graded alcohol solutions. For antigen retrieval, the sections were heated in 25 $\mathrm{mmol} / \mathrm{L}$ sodium citrate $(\mathrm{pH} 9)$ at $95^{\circ} \mathrm{C}$ for 15 minutes. The slides were treated with $3 \% \mathrm{H}_{2} \mathrm{O}_{2}$ to block endogenous peroxidase activity, and then incubated overnight at $4^{\circ} \mathrm{C}$ with primary antibodies (FASN, 1:100; L-FABP, 1:50; VEGF, 1:30). After that, the sections were incubated with HRP-conjugated anti-rabbit/mouse IgG for $30 \mathrm{~min}$ at room temperature, and DAB mix (ZLI-9032, ZSGB-BIO) was applied for staining. The percentage of staining-positive tumor cells was quantified under the microscope and the mean value of 5 randomly- selected fields were used for comparison.

\section{Statistical analysis}

Real-time PCR, MTT and wound healing assay were carried out in triplicate. The average of three experiments were used for statistical analysis and presented as mean \pm Standard Deviation. Data were analyzed by GraphPad Prism version 5.0 for Windows (GraphPad Software, San Diego, CA, USA). A Student's t-test was used to detect the difference between groups and a $p$ value less than 0.05 was considered to be statistically significant. 


\section{Results}

\section{Confirmation of MCF-7-MEK5 cell stability}

The MCF-7 cells exhibited a typical morphology of epithelial cells with intact cell-to-cell contact. In contrast, MCF-7-MEK5 cells exhibited mesenchyme cell morphology with a more dispersed colony appearance and incompletely defined colony borders, suggesting an EMT phenotype (Figure 1A). Result of wound healing assay showed that MCF-7-MEK5 cells exhibited higher migration ability compared to MCF-7 cells at 24 hours after wounding (Figure 1B). The migration index of MCF-7-MEK5 cells was significantly higher than that of MCF-7 cells (Figure 1C). As MEK5 is a stimulator of EMT, overexpression of MEK5 in MCF-7 cells resulted in a stable EMT characterized by increased expression of vimentin and decreased E-cadherin. RT-PCR results showed that MCF-7-MEK-5 cells had an increased level of MEK5 (Figure 1D). Expression of vimentin, a marker of mesenchyme cells, was enhanced in MCF-7-MEK5 cells. In contrast, mRNA and protein levels of E-cadherin, a typical epithelial cell marker, was reduced (Figure 1D). Quantitative results showed that
MEK5 increased by $132.5 \pm 22.4$ folds $(\mathrm{p}<0.05)$ and vimentin by $246.8 \pm 37.6$ folds ( $<<0.05$, Figure 1E), while the E-cadherin mRNA decreased by $0.0123 \pm 0.002$ folds $(p<0.05)$ in MCF-7-MEK5 cells. WB result further confirmed increased levels of MEK5 and vimentin proteins and decreased E-cadherin protein (Figure 1F). These results suggested that overexpression of MEK-5 in MCF-7 cells resulted in a stable EMT and increased migration ability of MCF-7-MEK5 cells.

\section{Overexpression of FASN increased levels of L-FABP, VEGF, and VEGFR-2 in MCF-7-MEK5 cells}

The RT-PCR result showed an increase of FASN mRNA in MCF-7-MEK5 cells (in vitro) and in the animal model (in vivo) injected with MCF-7-MEK5 cells (Figure 2A). The quantitative real-time PCR result revealed that the FSAN level increased by $53.24 \pm 5.74$ folds $(\mathrm{p}<0.05)$ in in vitro experiment and by $65.75 \pm 8.23$ folds $(p<0.05)$ in in vivo experiment (Figure 2B). WB result further confirmed the increased expression of FASN protein in both models associated with MCF-7-MEK5 cells (Figure 2C).

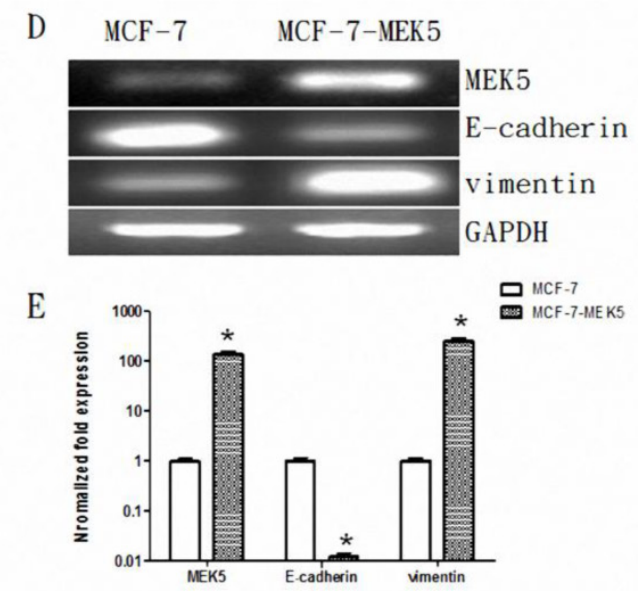

$\mathrm{F}$

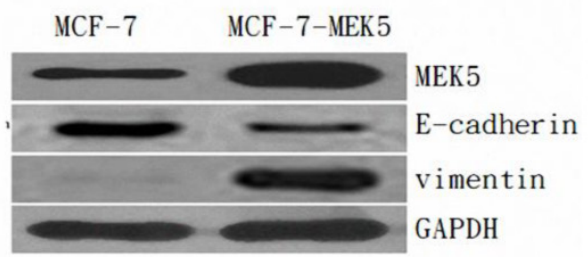

Figure I. Stability of MCF-7-MEK5 cells. A. Comparison of morphologic difference of MCF-7 and MCF-7-MEK5 cells. Compared to MCF-7 cells, MCF-7-MEK5 cells exhibited a more dispersed colony appearance and incompletely defined colony borders. B. Wound healing assay. MCF-7 (I, II) and MCF-7-MEK5 (III, IV) cells were cultured to form a confluent monolayer. Scratch wounds were made and photographs were taken immediately (I, III) or 24 h (II, IV) after wounding. C. Compared to MCF-7 cells, MCF-7-MEK5 cells showed stronger migration ability. D. RT-PCR assay was used to detect mRNA levels of MEK5, E-cadherin, and vimentin in both cells. E. Quantitative real-time PCR assay showed a decrease of E-cadherin and an increase of vimentin in MCF-7-MEK5 cells compared to MCF-7 cells. F. WB assay was used to detect protein levels of MEK5, E-cadherin, and vimentin in both cells. *p<0.05, $* * p<0.01$. 
A

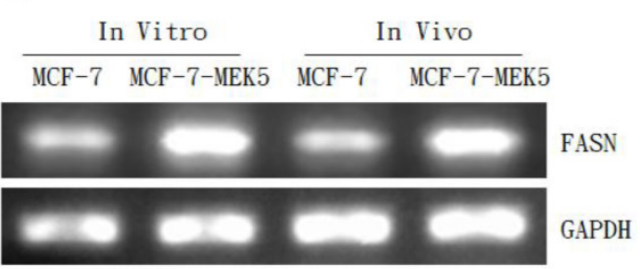

$\mathrm{B}$

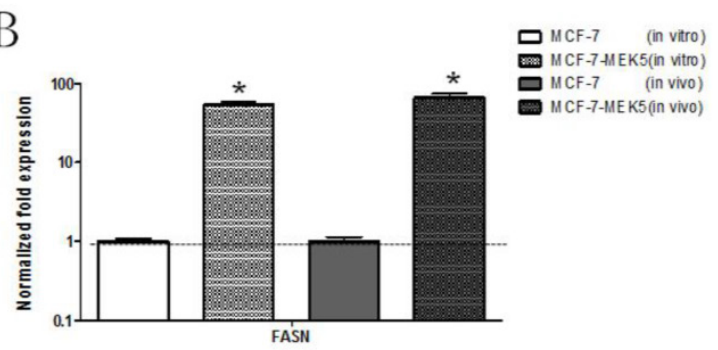

C

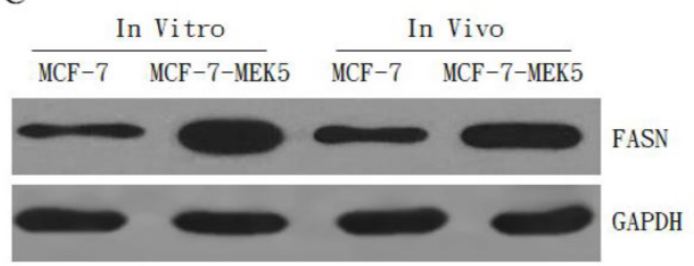

Figure 2. Expression levels of FASN increased in MCF-7-MEK5 cells and animal model injected with MCF-7-MEK5 cells. A. RT-PCR result showed increases of FASN mRNA level in both in vitro and in vivo models. B. Quantitative real-time PCR result revealed a significant increase of FASN mRNA in both models. C. WB showed an increase of FASN protein level. ${ }^{*} \mathrm{p}<0.05$.

A

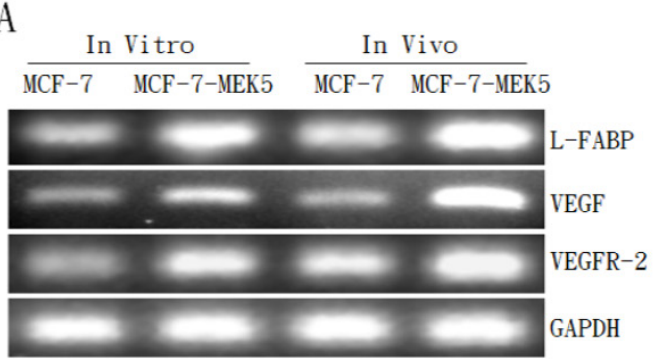

$\mathrm{C}$

$\frac{\text { In Vitro }}{\text { MCF-7 }} \quad$ MCF-7-MEK5 $5 \frac{\text { In Vivo }}{\text { MCF-7 MCF-7-MEK5 }}$

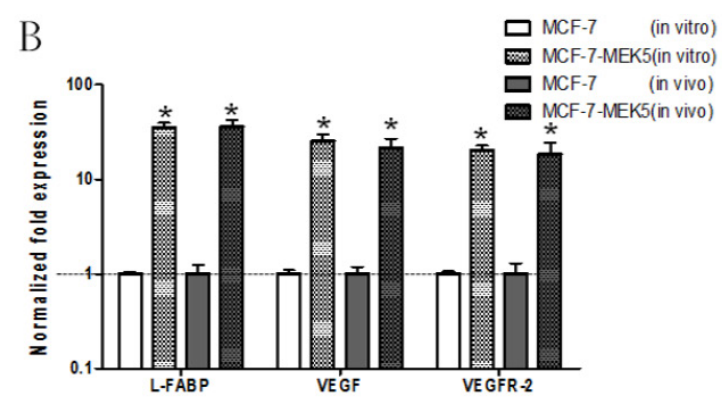

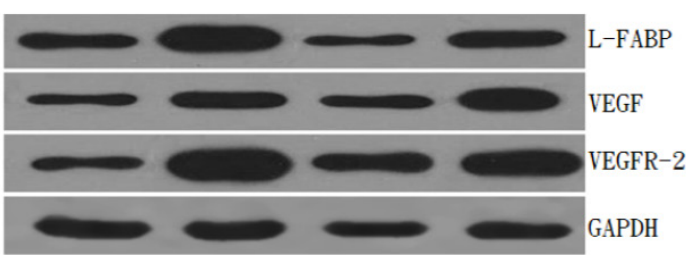

Figure 3. Expression levels L-FABP, VEGF, and VEGFR-2 increased in both in vivo and in vitro models with MCF-7-MEK5 cells. A. A representative RT-PCR result. B. Quantitative real-time PCR showed significant increases of L-FABP, VEGF and VEGFR-2 mRNA levels in both MCF-7-MEK5 cells and in nude mouse tumor tissues. C. WB assay showed increases of L-FABP, VEGF, and VEGFR-2 proteins. ${ }^{*} \mathrm{p}<0.05$.

Coincidently, mRNA levels of L-FABP, VEGF, and VEGFR-2 were also increased in both models associated with MCF-7-MEK5 cells (Figure 3A). Result of quantitative real-time PCR assay showed significant increases in three genes (Figure 3B). In MCF-7-MEK5 cells L-FABP increased by $45.58 \pm 3.97$ folds ( $\mathrm{p}<0.05)$, VEGF by $35.33 \pm 4.95$ folds $(\mathrm{p}<0.05)$, and VEGFR-2 by $30.28 \pm 2.53$ folds ( $p<0.05$ ). In nude mouse tumor tissues, L-FABP increased by $36.59 \pm 6.36$ folds $(\mathrm{p}<0.05)$, VEGF by $21.54 \pm 5.34$ folds $(\mathrm{p}<0.05)$, and VEGFR-2 by $18.46 \pm 5.75$ folds $(\mathrm{p}<0.05)$. WB result further revealed upregulations of L-FABP, VEGF, and VEGFR-2 proteins in both models with MCF-7-MEK5 cells (Figure 3C). These results suggested that upregulation of FASN was associated with increased expressions of FA binding protein L-FABP and VEGF and its receptor VEGFR-2. 


\section{Cerulenin inhibited MCF-7-MEK5 cells viability and migration}

We further used MTT assay to investigate the effect of FASN inhibitor, Cerulenin on the MCF-7-MEK5 cell viability. Cell morphology was observed at $0,12,20$, and 24 hours after $20 \mu \mathrm{g} / \mathrm{ml} \mathrm{Ce}-$ rulenin treatment (Figure 4A). When MEK5 was introduced into MCF-7 cells, MCF-7-MEK5 cells exhibited a decrease in cell-to-cell contact and an increase in cell migration ability that are features of EMT. After Cerulenin treatment, MCF-7-MEK5 cells exhibited classic epithelial cell morphology with increased cell-to-cell contact. This change was usually associated with mesenchymal-epithelial transition (MET), suggesting that inhibition of FASN could reverse EMT to MET. This result supported an important role of FASN in the EMT. Cell viability assay for MCF-7 and MCF-7-MEK5 cells were performed after 24-hour treatment with $0,5,10,20,40$, or $80 \mu \mathrm{g} / \mathrm{ml}$ Cerulenin (Figure 4B). Our result showed that MCF-7-MEK5 cells were more sensitive to Cerulenin than MCF-7 cells and exhibited a dramatic decrease in cell viability in response to $20 \mu \mathrm{g} / \mathrm{ml}$ Cerulenin.

To further study the effect of Cerulenin on MCF-7-MEK5 cell migration, we conducted wound healing assay in the presence of $15 \mu \mathrm{g} / \mathrm{ml}$ Cerulenin. Images were obtained from cells immediately after wounding (Figure 5A, I). Then cells were incubated with serum-free medium (II), medium with DMSO (III) or with Cerulenin $(15 \mu \mathrm{g} / \mathrm{ml}, \mathbf{I V})$ for 24 hours. Compared to the control and DMSO group, Cerulenin-treated group showed significantly decreased migration index (Figure 5B), suggesting that Cerulenin inhibited MCF-7-MEK5 cell migration effectively.
Moreover, quantitative real-time PCR assay showed that mRNA level of E-cadherin was increased significantly after Cerulenin treatment by $85.70 \pm 14.77$ folds (Figure $5 \mathrm{C}$ and D), whereas Cerulenin treatment decreased the expression of vimentin dramatically $(0.016 \pm 0.004, p<0.05)$. WB assay showed corresponding changes of E-cadherin and vimentin proteins (Figure 5E). This result suggested that inhibition of FASN by Cerulenin might decrease the migration and inhibit EMT in MCF-7-MEK5 cells.

\section{Inhibition of FASN with Cerulenin decreased expressions of L-FABP, VEGF, and VEGFR-2 in both in vitro and in vivo models with MCF-7-MEK5 cells}

To further study the inhibitory effect of Cerulenin, we examined both mRNA and protein levels of L-FABP, VEGF, and VEGFR-2 in the presence of Cerulenin. The PCR results showed that compared to the control group, the Cerulenin-treated group showed a decrease in FASN mRNA expression in both models (Figure 6A). Additionally, Cerulenin treatment also decreased mRNA levels of L-FABP, VEGF, and VEGFR-2 in both models. Quantitative RT-PCR results showed that the Cerulenin group had significantly lower level of FASN $(0.023 \pm 0.005, \mathrm{p}<0.05)$, L-FABP $(0.024 \pm 0.003, \mathrm{p}<0.05)$, VEGF $(0.038 \pm 0.004$, $\mathrm{p}<0.05)$, and VEGFR-2 $(0.027 \pm 0.004, \mathrm{p}<0.05)$ compared to the control group in in vitro experiment (Figure 6B). Similar results were also observed in the in vivo experiment. WB results further confirmed down-regulations of protein level of these genes (Figure 6C). This result suggested that the inhibitory effect of Cerulenin might be exerted through regulating expressions of FASN and its downstream pro-
A

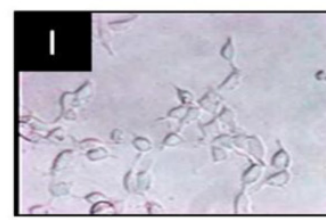

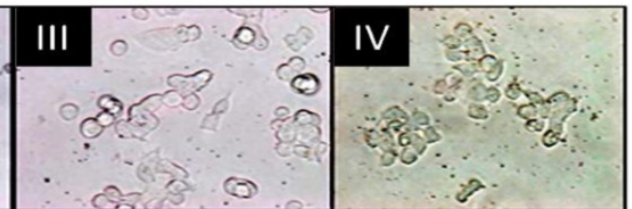

B

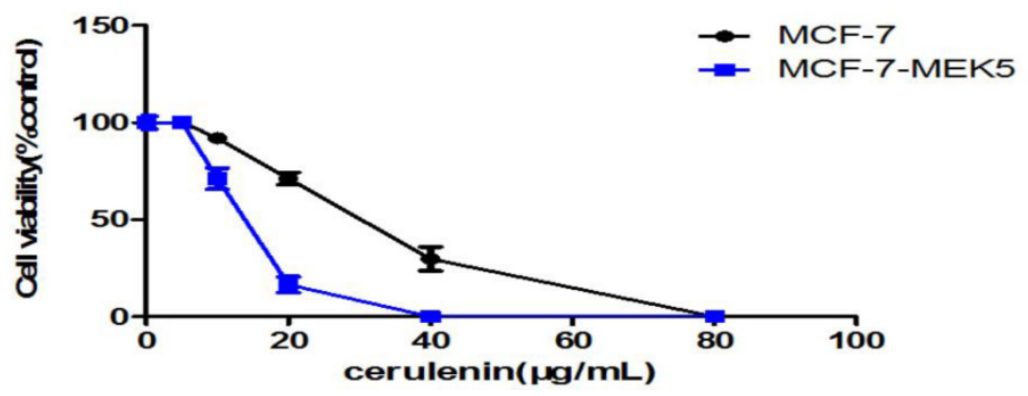

teins.

Figure 4. Cerulenin effectively inhibited MCF-7-MEK5 cell viability. A. Morphological changes of MCF-7-MEK5 cells were photographed at $0(\mathrm{I}), 12$ (II), 20 (III) and $24 \mathrm{~h}$ (IV) after Cerulenin $(20 \mathrm{\mu g} / \mathrm{ml})$ treatment. B. Cell viability assay. MCF-7 and MCF-7-MEK5 cells were pretreated with Cerulenin $(0,5,10,20,40$, and 80 $\mu \mathrm{g} / \mathrm{ml}$ ) for 24 hours followed by cell viability assay. This result showed that MCF-7-MEK5 cells were more MCF-7 cells. sensitive to Cerulenin than 
A

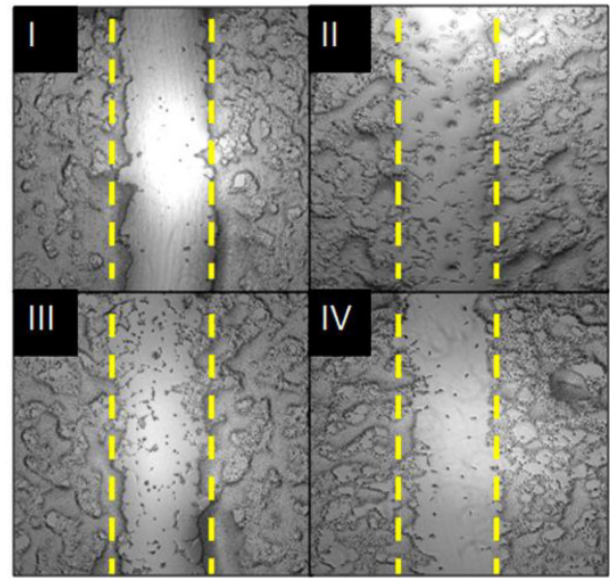

B

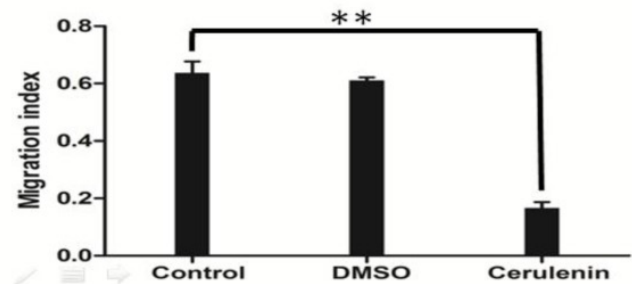

C

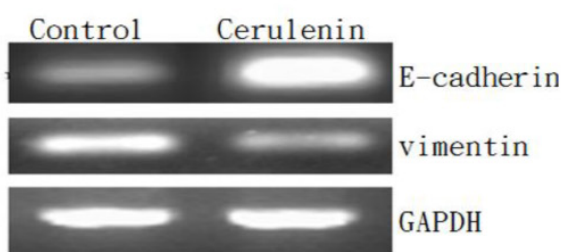

D

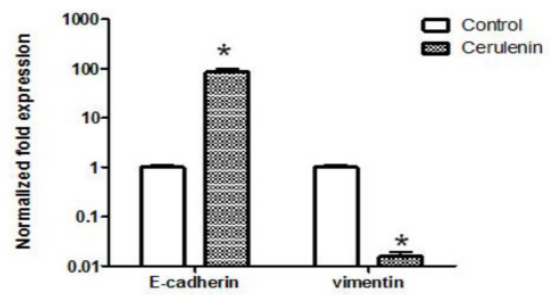

E

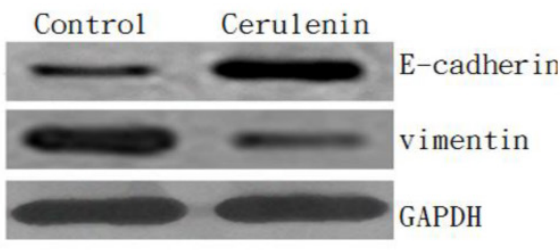

Figure 5. Cerulenin-treatment inhibited migration and EMT of MCF-7-MEK5 cells. A. Wound healing assay. MCF-7-MEK5 confluent monolayers were scratched and an image was taken immediately (I). Serum-free medium only (II, control), medium with DMSO (III), or with Cerulenin (I5 $\mu \mathrm{g} / \mathrm{ml}$, IV) were then added and incubated for $24 \mathrm{~h}$. B. The migration index of the Cerulenin group was significantly lower than the DMSO and control group $(\mathrm{p}<0.0 \mathrm{I})$, suggesting that Cerulenin inhibited MCF-7-MEK5 cell migration. C. Cerulenin treatment increased E-cadherin mRNA and decreased vimentin mRNA in MCF-7-MEK5 cells. D. Quantitative real-time PCR assay showed that MCF-7-MEK5 cells had increased E-cadherin by $85.69 \pm$ I 4.76 folds and decreased level of vimentin $(0.016 \pm 0.004)$. E. WB assay showed an increase in E-cadherin and a decrease in vimentin proteins after Cerulenin treatment. ${ }^{*} \mathrm{p}<0.05$, $* * \mathrm{p}<0.01$.
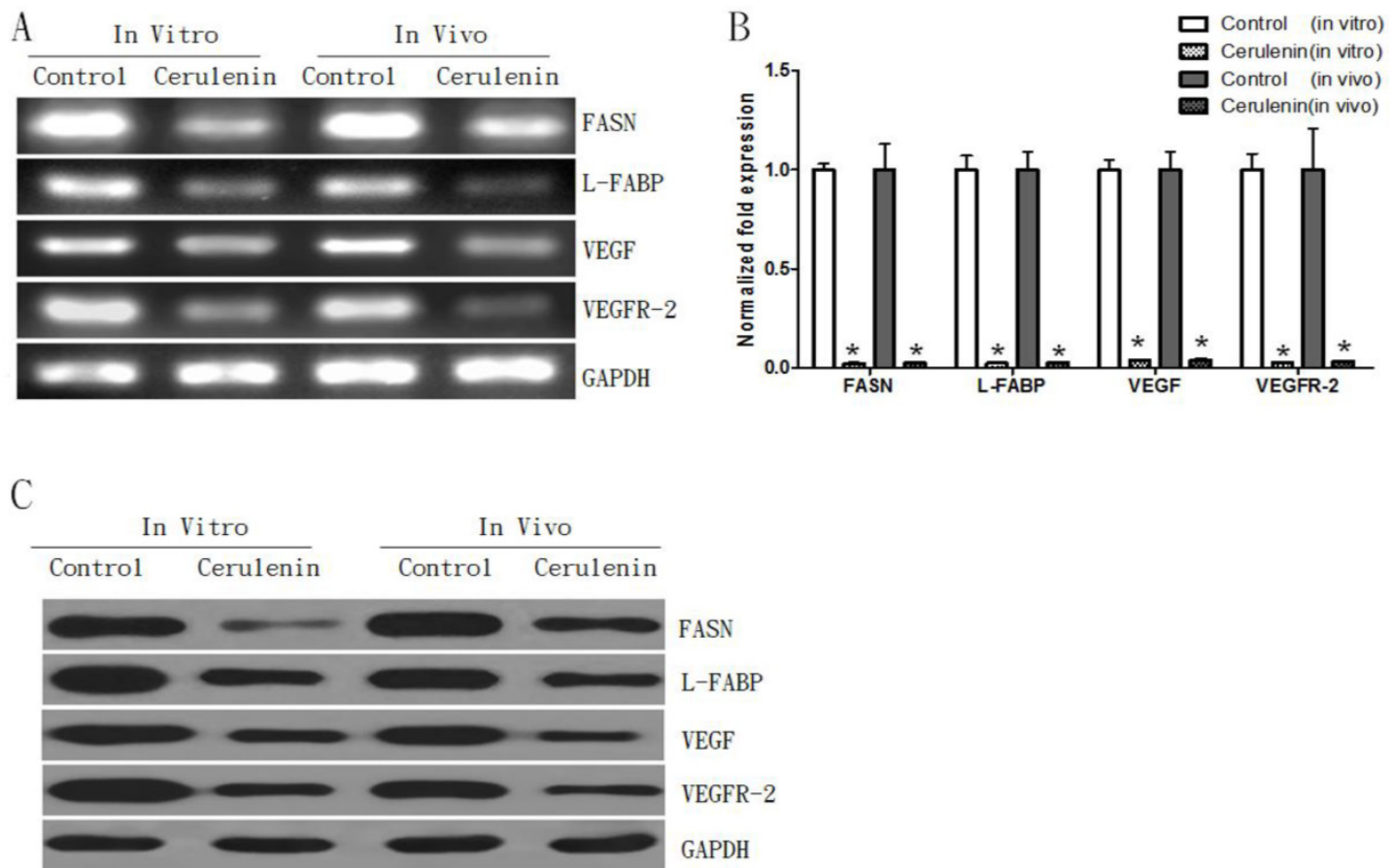

Figure 6. Inhibition of FASN decreased expressions of L-FABP, VEGF, and VEGFR-2 in both in vitro and in vivo models. A. RT-PCR result assay showed decreases of FASN, L-FABP, VEGF, and VEGFR-2 mRNAs in MCF-7-MEK5 cells. B. Quantitative real-time PCR assay showed that Cerulenin treatment significantly decreased levels of FASN, L-FABP, VEGF, and VEGFR-2 in MCF-7-MEK5 cells. In nude mouse tumor tissues (in vivo model), Cerulenin treatment also decreased levels of FASN, L-FABP, VEGF, and VEGFR-2. C. WB result showed that proteins of FASN, L-FABP, VEGF, and VEGFR-2 were downregulated in MCF-7-MEK5 cells after Cerulenin treatment. 


\section{Colocalization of FASN with L-FABP or VEGF in MCF-7 and MCF-7-MEK5 cells}

We further used immunofluorescence cytochemistry to investigate the localization of FASN (red in Figure 7 A1-D1) and its co-labeling with downstream molecules VEGF (green in Figure 7 A2 and B2) and L-FABP (green in Figure 7 C2 and D2) within MCF-7 (Figure 7 A1-5 and C1-5) and MCF-7-MEK5 cells (Figure 7 B1-5 and D1-5). In MCF-7-MEK5 cells, FASN-positive signaling located in the majority of the cell but nucleus (red in Figure 7 B1 and D1). VEGF staining (green in Figure 7B2) showed similar distribution pattern as FASN and co-localization of FASN and VEGF (yellow in Figure 7 B4 and B5) mainly located in most cell area except nucleus. L-FABP staining located in both cytoplasm and nucleus (green in Figure 7D2). Co-localization of FASN and L-FABP appeared on most cell area except nucleus (Figure 7 D4 and D5). Immunofluorescence staining results further showed that MCF-7-MEK5 cells had increased expression of FASN compared to MCF-7 cells (red in Figure 7 A1-D1). Additionally, VEGF (green in Figure 7B2) and L-FABP (green in Figure 7D2) in MCF-7-MEK5 cells were also higher than those in MCF-7 cells, suggesting that upregulation of FASN were associated with increased expression of its downstream proteins VEGF and L-FABP. Quantita- tive analysis was further used to compare colocalized signal between MCF-7-MEK5 and MCF-7 cells. FASN and VEGF colabeling intensity in MCF-7-MEK5 cells was significantly higher than that in MCF-7 cells $(78.73 \pm 4.64$ vs. $39.63 \pm 4.64, p<0.001)$. FASN and L-FABP colocalization in MCF-7-MEK5 was also significantly higher than that in the MCF-7 cells (69.66 \pm 3.69 vs. $31.67 \pm 3.79$, $p<0.001)$.

\section{Expressions of FASN, L-FABP and VEGF in nude mouse tumor tissues}

To evaluate the expressions of FASN, L-FABP and VEGF in tumor tissues, we performed immunohistochemistry assay and used the percentage of FASN-, L-FABP- or VEGF- positive cells as an index for the level of expression (Table 2). Quantitative results showed that the MFC-7-MEK5 group had significantly increased FASN-positive cells compared to the MCF-7 group $(p<0.001)$. Increased percentage of FASN-positive cells was accompanied by increased percentages of L-FABP- and VEGF-positive cells in the MCF-7-MEK5 group. Cerulenin treatment significantly decreased the percentage of FASN- L-FABPand VEGF-positive cells $(\mathrm{P}<0.001)$, suggesting that inhibition of FASN could reduce expression L-FABP and VEGF in tumor tissues.
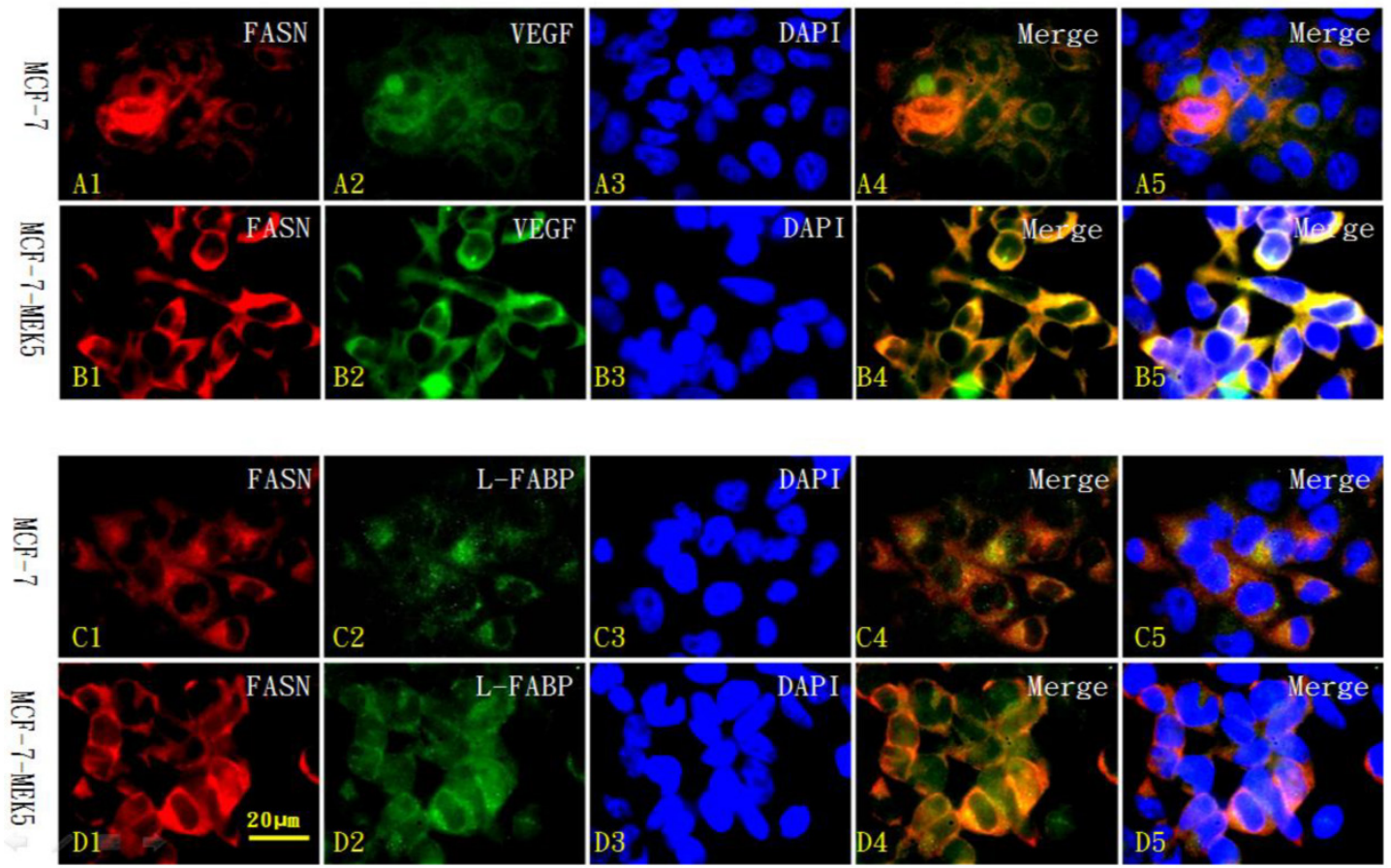

Figure 7. Immunofluorescence cytochemistry for localization of FASN with L-FABP or VEGF in MCF-7 and MCF-7-MEK5 cells. Localization of FASN (red) with VEGF (green) in MCF-7 cells (A) and MCF-7-MEK5 cells (B) were presented. DAPI staining was used to identify cell nuclei. Compared to the MCF-7 cells, MCF-7-MEK5 cells showed increased expression of FASN and VEGF and more co-labeling of two proteins (yellow) within cells except for nucleus. Localization of FASN (red) with L-FABP (green) in MCF-7 (C) and MCF-7-MEK5 cells (D) were presented. MCF-7-MEK5 cells showed higher level of L-FABP expression distributed in both cytoplasma and nucleus. Compared to the MCF-7 cells, MCF-7-MEK5 cells showed significant co-labeling of FASN and L-FABP (yellow) in most cell area except nucleus. Scale bar $=20 \mu \mathrm{m}$. 
Table 2. Comparisons of FASN-, L-FABP- and VEGF-positive cells in nude mouse tumor tissues $(x \pm s)$.

\begin{tabular}{llll}
\hline Antibodies & $\begin{array}{l}\text { MCF-7 group } \\
\text { (positive cells, \%) }\end{array}$ & $\begin{array}{l}\text { MCF-7-MEK5 } \\
\text { group (positive } \\
\text { cells, \%) }\end{array}$ & $\begin{array}{l}\text { MCF-7-MEK5/ } \\
\text { Cerulenin (positive } \\
\text { cells, \%) }\end{array}$ \\
\hline FASN & $12.93 \pm 2.41$ & $88.27 \pm 6.43^{* * *}$ & $27.42 \pm 2.08^{* * *}$ \\
L-FABP & $22.62 \pm 5.39$ & $87.17 \pm 6.02^{* * *}$ & $16.68 \pm 3.12^{* * *}$ \\
VEGF & $29.07 \pm 1.84$ & $85.22 \pm 7.93^{* * *}$ & $33.75 \pm 3.10^{* * *}$ \\
\hline
\end{tabular}

\section{Discussion}

Our results showed that FASN, L-FABP, VEGF, and VEGFR-2 were elevated in MCF-7-MEK5 cells compared to those in MCF-7 cells. Cerulenin, the potent FASN inhibitor, inhibits MCF-7-MEK5 cells proliferation and migration associated with EMT, and blocks the expressions of FASN, L-FABP, VEGF, and VEGFR-2. In addition, L-FABP and VEGF exhibited co-localization with FASN. All results suggest that FASN is essential to EMT and affects L-FABP, VEGF, and VEGFR-2 during EMT.

MCF-7 cells exhibit typical epidermal characteristics, round shape and cell-to-cell contact, and are prone to forming cell colony. When MEK5 was transferred into MCF-7 cells, MCF-7-MEK5 cells showed reduced cell-to-cell contact and decreased number in cell colonies, but increased migration capacity, suggesting an occurrence of EMT. EMT is characterized by decreased cell-cell contact, accompanied by decrease of E-cadherin and increase of vimentin. In contrast, MET is characterized by increased cell-cell contact, accompanied by increase of E-cadherin and decrease of vimentin. In this study, Cerulenin-treated MCF-7-MEK5 cells resumed epidermal cells (MCF-7) features, showing as increased cell contact and formation of colony. Additionally, increased E-cadherin and decreased vimentin were also observed in Cerulenin-treated MCF-7-MEK5, suggesting that inhibition of FASN by Cerulenin reversed EMT and promoted MET. Actually, specific knock-down of FASN in metastatic cells has been reported before by Gonzalez-Guerrico et al. [12]. In this study shRNA-mediated knock-down of FASN completely abrogated the anchorage-independent growth ability of the metastatic cells that reverted from EMT to MET. Combined with our finding, all these showed an essential role of FASN in EMT.

FASN is related to EMT in many cancers, including colorectal cancer ${ }^{[13]}$ and skin cancer ${ }^{[14]}$. These studies focused on the regulation of FASN on the activation of receptor tyrosine kinases (RTKs) localized on cell membrane, including Her2 [15], EGFR [16], and insulin-like growth factor-I receptor [17]. The ubiquitous effects of FASN on several RTKs suggest that
FASN might act on the plasma membrane-associated proteins by a more general mechanism.

VEGFR-2 is one of the receptors that interact with VEGF, which is correlated with metastasis and invasion in many tumors, including lung cancer [18], pancreatic cancer [19], and breast cancer [6]. Gonzalez et al. found that VEGF could induce EMT in prostate intraepithelial neoplasia cells [5]. Few studies have explored the correlation between FASN and VEGF, yet the results are debatable. Selvendiran et al. reported that FASN inhibitor HO-3867 could effectively inhibit the metastasis and invasion of human ovarian cancer cells A2780 and SKOV3 through the inhibition of VEGF expression [20]; however, Menendez et al. reported that in breast cancer cells BT474, VEGF was elevated via MAPK signaling pathway after FASN was inhibited by C75 [15].

Fatty acids synthesized by FASN can be transferred to the cell membrane or intracellular organelles to form the plasma membrane or provide energy. They play an important role in almost a whole cell [21]. L-FABP has been hypothesized to be involved in lipid absorption by the enterocyte, and in hepatocyte lipid transport and lipoprotein metabolism. L-FABP transports many ligands, yet this transportation is more predisposed to long chain saturated fatty acids, the main products of FASN [22]. In addition, L-FABP deficiency can protect mice from the fatty liver induced by long-term high fat diet, yet such protection is only effective for saturated fatty acids, and benumbed with unsaturated fatty acids [23]. All these suggest a correlation of L-FABP and FASN. Besides, L-FABP involves in partitioning FA to specific lipid metabolic pathways. Correspondingly, L-FABP impacts orientation of cell membrane localized protein, and hence influences functions of these proteins [7]. Meanwhile, L-FABP is co-localized with VEGF in lung cancer [24], and liver cancer cells [25]. All these suggest that FASN may regulate VEGF and VEGFR-2 via L-FABP.

In summary, the mechanism of FASN inducing the EMT in MCF-7-MEK5 cells may be via the following pathway: FASN over-expression results in the accumulation of long-chain saturated fatty acids, which are transferred to the cell membrane by L-FABP. VEGFR-2 localization is regulated by L-FABP, and its ligand VEGF is activated correspondingly to induce EMT.

Besides, MCF-7-MEK5 cells exhibit TNF-a resistance. However, Cerulenin could effectively inhibit MCF-7-MEK5 viability. This suggests that FASN inhibition could be conducted as individual therapies to patients with TNF-a resistance. 


\section{Acknowledgements}

The authors thank the Immunology Laboratory of Sichuan University for supplying breast cancer cells, and thank the National Key Laboratory of Biological Treatment for equipment support. This work was supported by the National Natural Science Foundation of China (NSFC, J1103604 and 30870688). The founders had no role in study design, data collection and analysis, decision to publish, or preparation of the manuscript.

\section{Abbreviations}

$\mathrm{DAB}, 3,3$ '-diaminobenzidine; DAPI, 2-(4-Amidinophenyl)-6-indolecarbamidine dihydrochloride; DMSO, dimethyl sulfoxide; EMT, epithelial-mesenchymal transition; MET, mesenchymal-epithelial transition; FASN, fatty acid synthase; L-FABP, liver fatty acid binding protein; MTT, 3-(4,5)-dimethylthiahiazo (-z-y1)-3,5-di- phenytetrazoliumromide; PIN, prostateintraepithelialneoplasia; VEGF, vascular endothelial growth factor; VEGFR-2, vascular endothelial growth factor receptor-2; MEK5, mitogen-activated protein kinase (MAPK) kinase 5; TNF-a, tumor necrosis factor-alpha.

\section{Competing Interests}

The authors have declared that no competing interest exists.

\section{References}

1. Drasin DJ, Robin TP, Ford HL. Breast cancer epithelial-to-mesenchymal transition: examining the functional consequences of plasticity. Breast Cancer Research. 2011; 13:226-38.

2. Sampaio JL, Gerl MJ, Klose C, et al. Membrane lipidome of an epithelial cell line. Proc Natl Acad Sci U S A. 2011; 108:1903-7.

3. Hung CM, Kuo DH, Chou $\mathrm{CH}$, et al. Osthole suppresses Hepatocyte growth Factor (HGF)-Induced Epithelial-Mesenchymal Transition via repression of the c-Met/Akt/mTOR Pathway in Human Breast Cancer Cells. J Agric Food Chem. 2011; 59:9683-90.

4. Kerbel RS, Kamen BA. The anti-angiogenic basis of metronomic chemotherapy. Nat Rev Cancer. 2004; 4:423-36.

5. Gonzalez-Moreno O, Lecanda J, Green JE, et al. VEGF elicits epithelial-mesenchymal transition (EMT) in prostate intraepithelial neoplasia (PIN)-like cells via an autocrine loop. Exp Cell Res. 2010; 316:554-67.

6. Mercurio AM, Lipscomb EA, Bachelder RE. Non-Angiogenic Functions of VEGF in Breast Cancer. J Mammary Gland Biol Neoplasia. 2005; 10:283-90.

7. Storch J, Thumser AE. The fatty acid transport function of fatty acid-binding proteins. Biochim Biophys Acta. 2000; 1486:28-44.

8. Storch J, Corsico B. The emerging functions and mechanisms of mammalian fatty acid-binding proteins. Annu Rev Nutr. 2008; 28:73-95.

9. Tun HW, Marlow LA, von Roemeling CA, et al. Pathway Signature and Cellular Differentiation in Clear Cell Renal Cell Carcinoma. Plos one. 2010; 5:e10696.

10. Changhua Zhou, Ashley M Nitschke, Wei Xiong et al. Proteomic analysis of tumor necrosis factor- $\alpha$ resistant human breast cancer cells reveals a MEK5/Erk5-mediated epithelial-mesenchymal transition phenotype. Breast Cancer Research. 2008; 10: R105.

11. Menendez JA, Vellon L, Lupu R. Targeting fatty acid synthase-driven lipid rafts: a novel strategy to overcome trastuzumab resistance in breast cancer cells. Med Hypotheses. 2005; 64: 997-1001.

12. A . Gonzalez-Guerrico and R. Lupu. Fatty Acid Synthase (FASN) as a Therapeutic Target for Breast Cancer Metastasi. Cancer Res 2009; 69 (24 Suppl): Abstract nr 6160 .

13. Zaytseva YY, Rychahou PG, Gulhati P, et al. Inhibition of Fatty Acid Synthase Attenuates CD44-Associated Signaling and Reduces Metastasis in Colorectal Cancer. Cancer Res. 2012; 72: 1504-17.

14. Kapur P, Rakheja D, Roy LC, et al. Fatty acid synthase expression in cutaneous melanocytic neoplasms. Mod Pathol. 2005; 18:1107-12.
15. Menendez JA, Vellon L, Mehmi I, et al. Inhibition of fatty acid synthase (FAS) suppresses HER2neu (erbB-2) oncogene overexpression in cancer cells. Proc Natl Acad Sci U S A. 2004; 101:10715-20.

16. Adachi S, Nagao T, To S, et al. (-)-Epigallocatechin gallate causes internalization of the epidermal growth factor receptor in human colon cancer cells. Carcinogenesis. 2008; 2910: 1986-93.

17. Shimizu M, Deguchi A, Hara Y, et al. EGCG inhibits activation of the insulin-like growth factor-1 receptor in human colon cancer cells. Biochem Biophys Res Commun. 2005; 334: 947-53.

18. Chen CH, Lai JM, Chou TY, et al.. VEGFA Upregulates FLJ10540 and Modulates Migration and Invasion of Lung Cancer via PI3K/AKT Pathway. PLoS One. 2009; 4 :e5052.

19. Yang AD, Camp ER, Fan F, et al. Vascular Endothelial Growth Factor Receptor-1 Activation Mediates Epithelial to Mesenchymal Transition in Human Pancreatic Carcinoma Cells. Cancer Res. 2006; 66: 46-51.

20. Selvendiran K, Ahmed S, Dayton A, et al. HO-3867, a synthetic compound, inhibits migration and invasion of ovarian carcinoma cells through downregulation of fatty acid synthase and focal adhesion kinase. Mol Cancer Res. 2010; 8: $188-97$.

21. Kuhajda FP. Fatty-acid synthase and human cancer: new perspectives on its role in tumor biology. Nutrition. 2000; 16: 202-8.

22. Storch J, McDermott L. Structural and Functional Analysis of Fatty Acid-Binding Proteins. J Lipid Res. 2009; 50 (Suppl): S126-31.

23. Newberry EP, Kennedy SM, Xie $Y$, et al. Diet-induced obesity and hepatic steatosis in L-Fabp(-/-) mice is abrogated with SF, but not PUFA, feeding and attenuated after cholesterol supplementation. Am J Physiol Gastrointest Liver Physiol. 2008; 294:G307-14.

24. Kawamura T, Kanno R, Fujii $\mathrm{H}$, et al. Expression of Liver-Type Fatty-Acid-Binding Protein, Fatty Acid Synthase and Vascular Endothelial Growth Factor in Human Lung Carcinoma. Pathobiology. 2005; 72:233-40

25. Dong LH, Li H, Wang F, et al. Expression of liver-type fatty acid-binding protein and vascular endothelial growth factor and their correlation in human hepatocellular carcinoma. Journal of Southern Medical University. 2007; 27:318-321. 